\title{
VE-cadherin RGD motifs promote metastasis and constitute a potential therapeutic target in melanoma and breast cancers
}

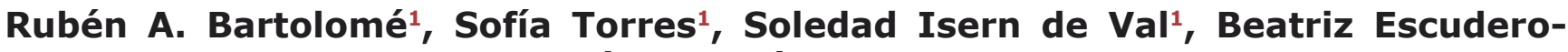 \\ Paniagua $^{1}$, Eva Calviño ${ }^{1}$, Joaquín Teixidó ${ }^{1}$ and J. Ignacio Casal $^{1}$ \\ ${ }^{1}$ Department of Cellular and Molecular Medicine, Centro de Investigaciones Biológicas (CIB-CSIC), Ramiro de Maeztu, \\ Madrid, Spain \\ Correspondence to: J. Ignacio Casal, email: icasal@cib.csic.es
}

Keywords: VE-cadherin, RGD motif, metastasis, melanoma, breast cancer

Received: November 08, 2016 Accepted: November 14, 2016

Published: December 09, 2016

\section{ABSTRACT}

We have investigated the role of vascular-endothelial (VE)-cadherin in melanoma and breast cancer metastasis. We found that VE-cadherin is expressed in highly aggressive melanoma and breast cancer cell lines. Remarkably, inactivation of VEcadherin triggered a significant loss of malignant traits (proliferation, adhesion, invasion and transendothelial migration) in melanoma and breast cancer cells. These effects, except transendothelial migration, were induced by the VE-cadherin RGD motifs. Co-immunoprecipitation experiments demonstrated an interaction between VE-cadherin and a2 $\beta 1$ integrin, with the RGD motifs found to directly affect $\beta 1$ integrin activation. VE-cadherin-mediated integrin signaling occurred through specific activation of SRC, ERK and JNK, including AKT in melanoma. Knocking down VEcadherin suppressed lung colonization capacity of melanoma or breast cancer cells inoculated in mice, while pre-incubation with VE-cadherin RGD peptides promoted lung metastasis for both cancer types. Finally, an in silico study revealed the association of high VE-cadherin expression with poor survival in a subset of melanoma patients and breast cancer patients showing low CD34 expression. These findings support a general role for VE-cadherin and other RGD cadherins as critical regulators of lung and liver metastasis in multiple solid tumours. These results pave the way for cadherin-specific RGD targeted therapies to control disseminated metastasis in multiple cancers.

\section{INTRODUCTION}

Metastasis, the final step of malignant transformation, is a complex process with numerous distinct and sequential steps. Organ-specific metastasis depends on different molecules, including growth factors, receptors, proteases, chemokines and cellular adhesion molecules, such as cadherins and integrins [1,2]. Recently, we described the presence of tripeptide RGD motifs in 7D cadherins, cadherin-17 (CDH17; also LI-cadherin) and cadherin-16, and type II cadherins, such as vascular endothelial (VE)-cadherin (CD144), KSP-cadherin (CDH6) and cadherin 20 (CDH20) [3]. CDH17 activates the $\alpha 2 \beta 1$ integrin pathway through specific RGD motifs to promote cell adhesion, proliferation and liver colonization in colorectal cancer metastasis [3, 4]. A direct interaction between $\mathrm{CDH} 17$ and $\alpha 2 \beta 1$ integrin was demonstrated using various methods, such as affinity chromatography, soluble binding of cadherin ectodomains and cell adhesion to RGD-containing cadherin domains [3].

VE-cadherin is a type II cadherin that contains RGD motifs within different domains (e.g. 1, 2 or 3) in many mammals and birds, but not in rodents or dogs (Supplementary Data Figure 1). In primates, the VEcadherin sequence displays two RGD motifs in cadherin domains 2 and 3 (Supplementary Data Figure 1). VEcadherin is mainly expressed in endothelial cells, where it plays a central role in vascular integrity and permeability and promotes homotypic cell-to-cell adhesion [5]. Intracellular association to $\beta$ - and $\gamma$-catenin (plakoglobin) promotes binding to the actin cytoskeleton [6]. Mice deficient in VE-cadherin, or expressing truncated VEcadherin, die in mid-gestation from severe vascular defects, involving endothelial apoptosis and disrupted survival signalling pathways [7]. VE-cadherin has been used as a target to control tumour angiogenesis 
in vivo [8]. It is also expressed in Ewing sarcoma [9], highly aggressive cutaneous melanomas [10] and it is involved in vasculogenic mimicry (the ability to form novel blood vessel-like structures) in uveal melanomas [11]. VE-cadherin is also expressed in a subset of acute lymphoblastic leukemia cells, where contributes to cell survival [12], and in a subset of cancer stem cells CD133+ in osteosarcoma, ovarian cancer and glioblastoma, contributing to vasculogenic mimicry by VEGFindependent tumour cell differentiation [13].

VE-cadherin enhances the capacity of mouse mammary tumour cells to proliferate and adhere to endothelial cells $[14,15]$. Its effects on cell proliferation have been commonly attributed to $\beta$-catenin release from the p120-catenin complex and induction of transcription of specific genes [16]. In addition, $\alpha 2 \beta 1$ integrinmediated phosphorylation of VE-cadherin leads to the disorganization of the endothelial adherens junctions and facilitates transendothelial migration of breast cancer cells. This effect was mediated by the binding of $\alpha 2 \beta 1$ integrin to an unknown counterligand on endothelial cells [17]. Still, many aspects regarding signalling effects of VEcadherin on the epithelial cancer cells and their effect on invasion and proliferation were still obscure.
Here, we investigated the role of VE-cadherin in metastasis progression of melanoma and breast cancers. We have identified that VE-cadherin activates $\alpha 2 \beta 1$ integrin through its RGD motifs, thereby using the integrin signalling pathway to promote adhesion, invasion and proliferation. We provide evidence that the VE-cadherin RGD motifs promote in vivo lung metastasis in melanoma and breast cancers. Moreover, a high expression of VE-cadherin in melanoma and breast cancer patients is associated to poor prognosis.

\section{RESULTS}

\section{VE-cadherin expression is associated to metastatic melanoma and breast cancer cell lines}

We analysed VE-cadherin expression and its role in metastasis in a panel of 8 melanoma and 4 breast cancer cell lines (Figure 1a). Compared to non-invasive MCF7, VE-cadherin overexpression was observed in metastatic breast cancer cells (SKBR3, MDA-MB-231 and MDAMB-468) and metastatic melanoma cell lines (BLM,
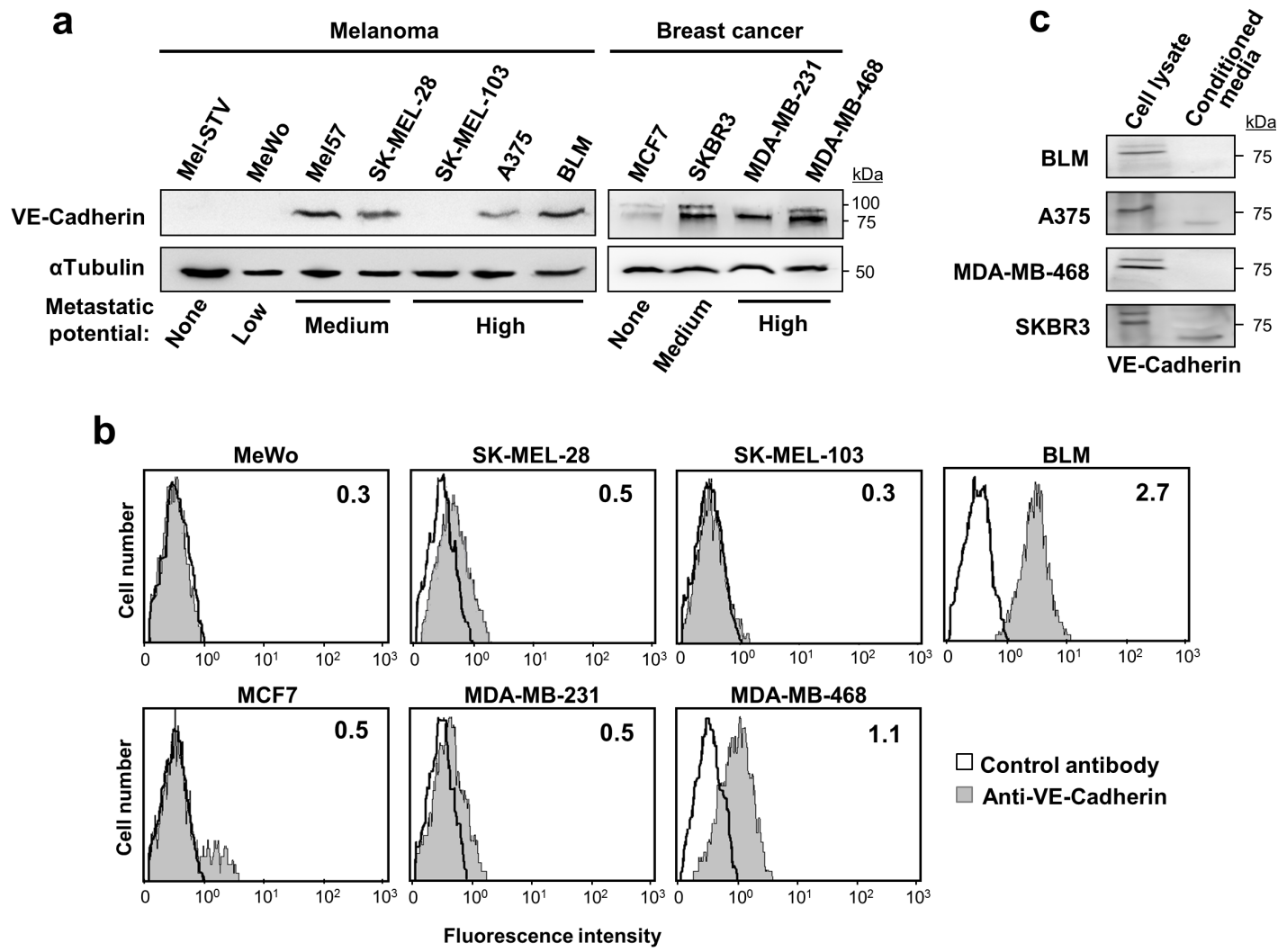

Figure 1: VE-cadherin is expressed in melanoma and breast cancer cell lines. a. Protein lysates of the indicated melanoma and breast cancer cell lines were resolved by SDS-PAGE and subjected to western blot using anti-VE-cadherin. Anti- $\alpha$-tubulin was used to assess total protein content. b. Flow cytometry analysis showing the surface expression of VE-cadherin on the indicated cancer cells. Inside each panel, mean fluorescence intensity is indicated. c. VE-cadherin expression was assessed by western blot in cell lysates and 48-h conditioned media of the indicated melanoma and breast cancer cell lines. 
A375, SK-MEL-28 and Mel57) except SK-MEL-103. VE-cadherin was absent in Mel-STV melanocytes and in the poorly metastatic MeWo cell line. Interestingly, breast cancer cells exhibited two forms of VE-cadherin while melanoma cells showed only one, probably due to cell-specific differences in glycosylation. Flow cytometry analysis confirmed a correlation between cell surface expression of VE-cadherin and total expression observed by western blot (Figure 1b). Secretion of the VE-cadherin ectodomain was detected in A375 melanoma cancer cells and SKBR3 breast cancer cells (Figure 1c). In any case, $75 \%$ of the cadherin was found in the cell lysate, indicating that VE-cadherin signalling would be mainly from the cell surface. For the remaining studies, two representative metastatic cell lines for each cancer type -BLM and A375 for melanoma and MDA-MB-468 and SKBR3 for breast cancer-were used.

\section{RGD peptides in VE-cadherin increase adhesion, proliferation and invasion in melanoma and breast cancer cells}

Next, we investigated whether VE-cadherin silencing affects the pro-metastatic properties of melanoma and breast cancer cells. Cancer cells transfected with either one of two distinct VE-cadherin siRNAs exhibited a clear reduction of VE-cadherin expression by western blot and flow cytometry (Figure 2a and Supplementary Data Figure 2a). VE-cadherin silencing led to a significant decline in the cellular responses of proliferation, invasion, transendothelial migration and adhesion (Figure $2 b$ and Supplementary Data Figure 2b). Flow cytometry analysis using a monoclonal antibody specific for the highaffinity conformation of $\beta 1$ integrin demonstrated that VE-cadherin silencing in the four cell lines significantly reduced the high affinity conformation (Figure $2 \mathrm{c}$ and Supplementary Data Figure 2c), but had no effect on the total level of $\beta 1$ integrin expression (Supplementary Data Figure 3), indicating that VE-cadherin regulates the affinity but not the expression of $\beta 1$ integrin. We thus investigated whether these effects are mediated through the VE-cadherin RGD motifs.

Addition of VE-cadherin RGD peptides significantly increased the activation of $\beta 1$ integrin (Figure $2 d$ ), as well as the adhesion, invasion and proliferation responses in melanoma and breast cancer cells (Figure 2e). With respect to the adhesion response, VE-cadherin increased cell binding to collagen type I and Matrigel (Figure 2e). In contrast, the RGD peptides did not affect the transendothelial migration capacity of tumour cells across an HUVEC endothelial cell monolayer, perhaps because the abundant expression of VE-cadherin in HUVEC tampers the peptide effects, or perhaps because the RGD motif does not participate in homotypic cellto-cell interactions [3]. Collectively, these data indicate that the VE-cadherin RGD motifs play a major role in cell adhesion, invasion and proliferation in melanoma and breast cancer cells, which correlate directly with $\beta 1$ integrin activation. The effect of VE-cadherin on transendothelial migration is likely mediated by homotypic interactions.

\section{VE-cadherin protein complexes are linked to integrin signalling and cell adhesion}

In contrast to $\mathrm{CDH} 17, \mathrm{VE}-$ cadherin contains a large cytoplasmic domain engaged in signal transmission. To characterize the protein-interaction network of VE-cadherin, we used immunoprecipitation and mass spectrometry. After bioinformatic analysis, we identified 79 proteins that co-immunoprecipitated specifically with VE-cadherin in BLM melanoma cells, and 24 proteins in MDA-MB-468 breast cancer cells (Supplementary Data Figure 4a and Table 2). The VE-cadherin interaction network in both cell types contained $\alpha 2 \beta 1$ integrin, $\alpha \mathrm{V}$ integrin and other proteins mainly involved in cell-matrix adhesion, cell-cell adhesion, cell signalling and the actin cytoskeleton (Figure 3a and Supplementary Data Figure $4 \mathrm{~b})$. On the other hand, we confirmed the interaction of VE-cadherin with $\mathrm{p} 120$-catenin and plakoglobin through the cytoplasmic domain [16]. In BLM melanoma cells, additional interactions with ALCAM, associated with high invasiveness and aggressiveness in melanomas, were also observed [18]. Among other VE-cadherinassociated proteins involved in cell signalling, survival and proliferation, we identified FAK, SRC, Ras, AXL, EPHA2, AKT, STAT1, STAT3, JNK and ERK kinases (Figure 3a). Although the number of interacting proteins was larger in melanomas than in breast cancer cells, many identified proteins were similar and functionally equivalent in both cancer types. Therefore, VE-cadherin has homologous protein networks in melanoma and breast cancer. Interaction between VE-cadherin and integrin may occur in trans (Figure 3a) and/or cis (Supplementary Data Figure 4c), as experimental data are compatible with both options. VE-cadherin-associated proteins were confirmed by western blot in the four cell lines, with only a few exceptions (lack of Ras in A375 and SKBR3 cells) (Figure $3 b)$.

The association of VE-cadherin with $\alpha 2 \beta 1$ integrin, and the presence of $\alpha \mathrm{V}$ integrin in the complex, were confirmed by combined co-immunoprecipitation and silencing studies (Figure 3c, 3d). $\alpha 2 \beta 1$ integrin interacts with VE-cadherin, as $\alpha 2$ integrin silencing caused the loss of VE-cadherin in the immunoprecipitate (Figure $3 \mathrm{~d}$ and Supplementary Data Figure 5). In contrast, $\alpha \mathrm{V}$ silencing did not change the levels of VE-cadherin. Still, we cannot exclude that $\alpha \mathrm{V}$ associates with $\beta 1$, or other integrin $\beta$ subunits, and clusters together with the $\alpha 2 \beta 1$ integrin. To investigate any potential effects of $\alpha \mathrm{V} \beta 1$ integrin, we carried out $\alpha \mathrm{V}$ and $\alpha 2$ integrin silencing in combination 

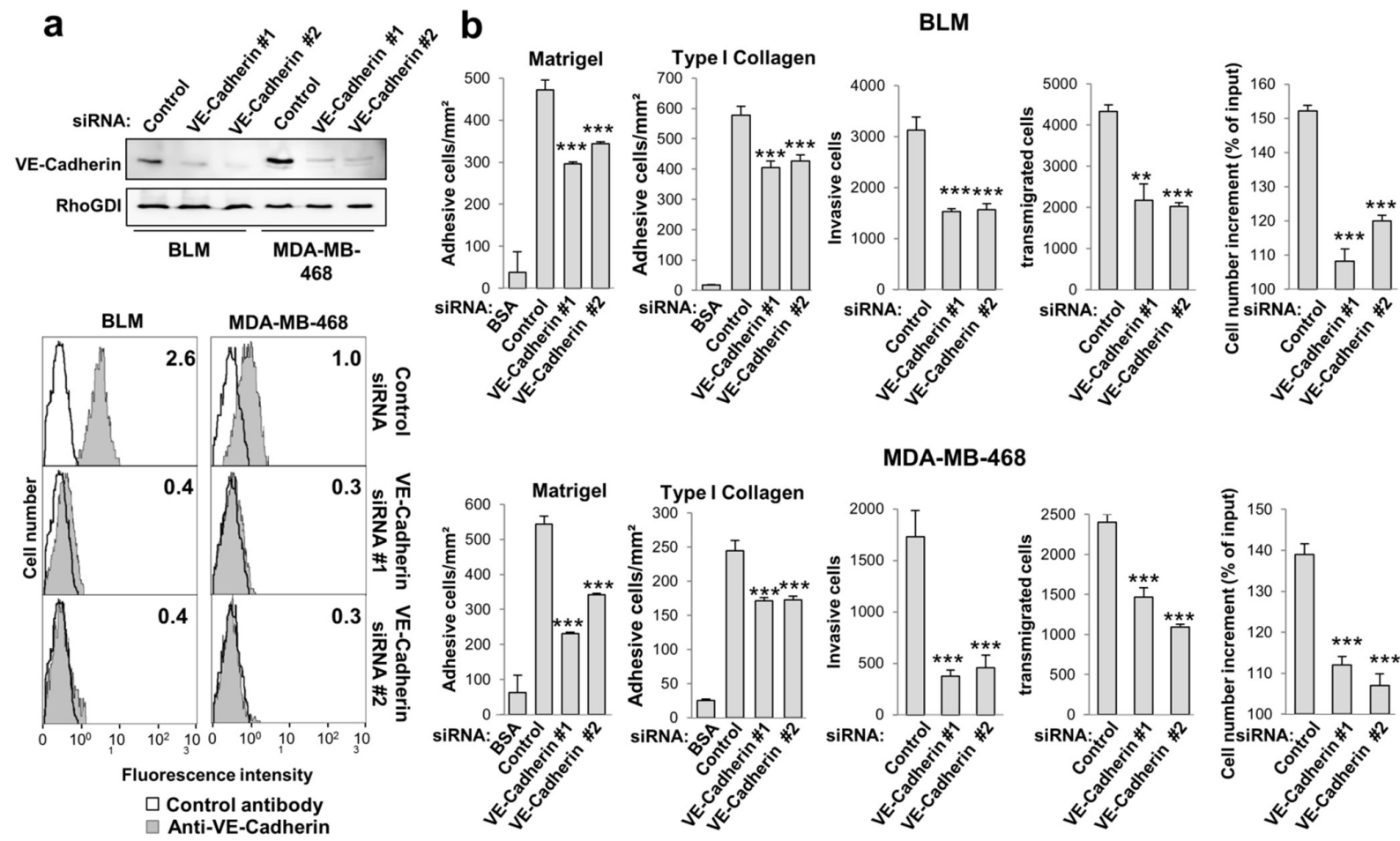

\section{MDA-MB-468}
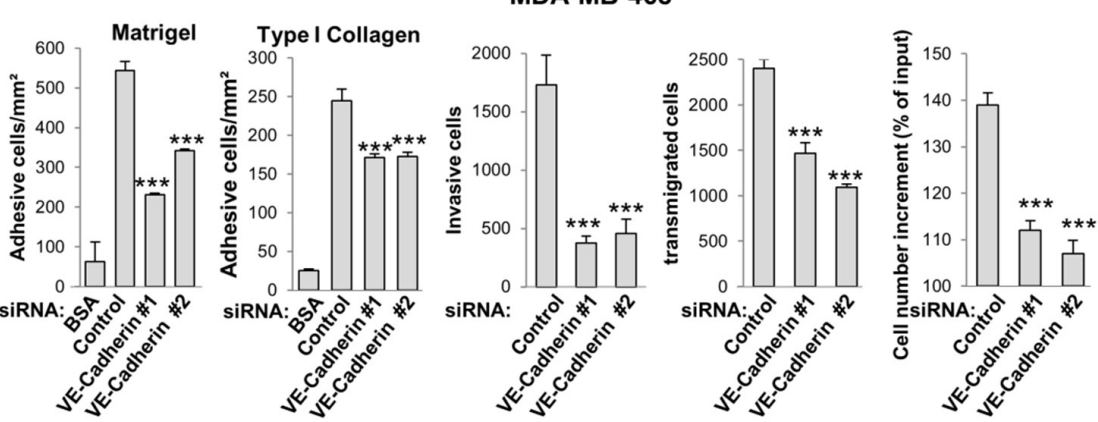

C

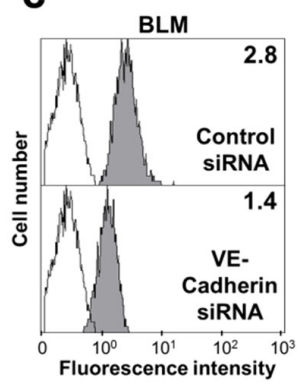

d

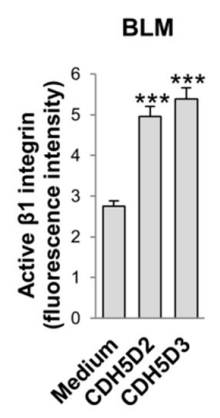

e
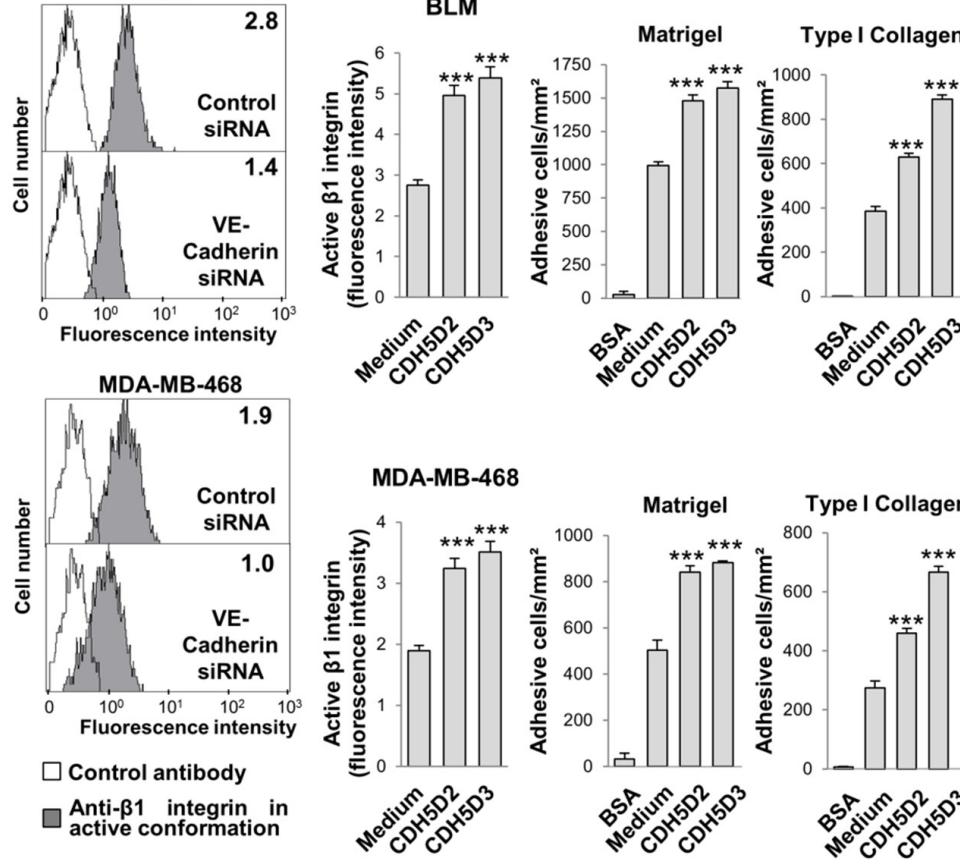

BLM
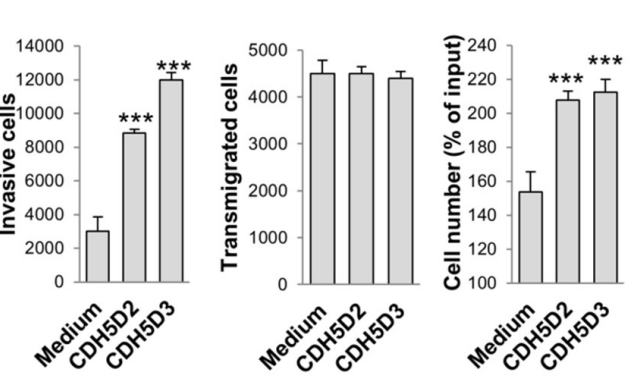

MDA-MB-468
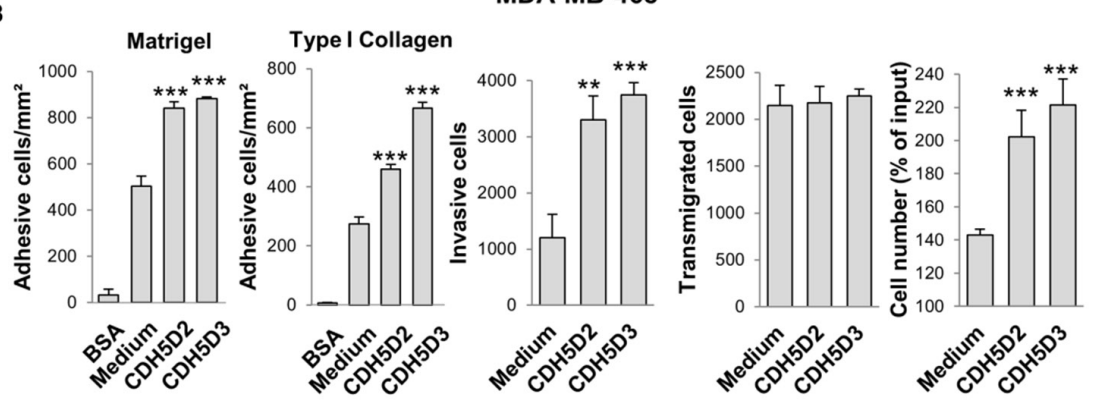

Figure 2: VE-cadherin RGD motifs promote cell adhesion, invasion and proliferation. a. BLM and MDA-MB-468 cells were transfected with one of two distinct siRNAs against VE-cadherin or with a control siRNA. VE-cadherin expression was assessed by western blot and flow cytometry. b. Transfectants were subjected to adhesion onto Matrigel or type I collagen, invasion through Matrigel, endothelial transmigration and MTT assays to determinate cell proliferation. Cell adhesion, invasion, transmigration or proliferation was significantly inhibited by VE-cadherin knock-down $(* * P<0.01, * * * P<0.001)$. c. Transfectants were analysed by flow cytometry to determine the expression of high-affinity $\beta 1$ integrin conformation. d. BLM and MDA-MB-468 cells were exposed to 9-mer peptides containing the RGD motifs and flanking sequences from VE-cadherin domain 2 or domain 3 (CDH5D2 or CDH5D3, respectively) and subjected to flow cytometry assays to assess the amount of $\beta 1$ integrin in high affinity conformation. $\beta 1$ integrin was significantly activated by exposition to the peptides (*** $P<0.001)$. e. Same as b. but in the presence of the CDH5D2 or CDH5D3 peptide. Cell adhesion, invasion or proliferation was significantly enhanced by peptide addition $(* * P<0.01$, *** $P<0.001)$. 


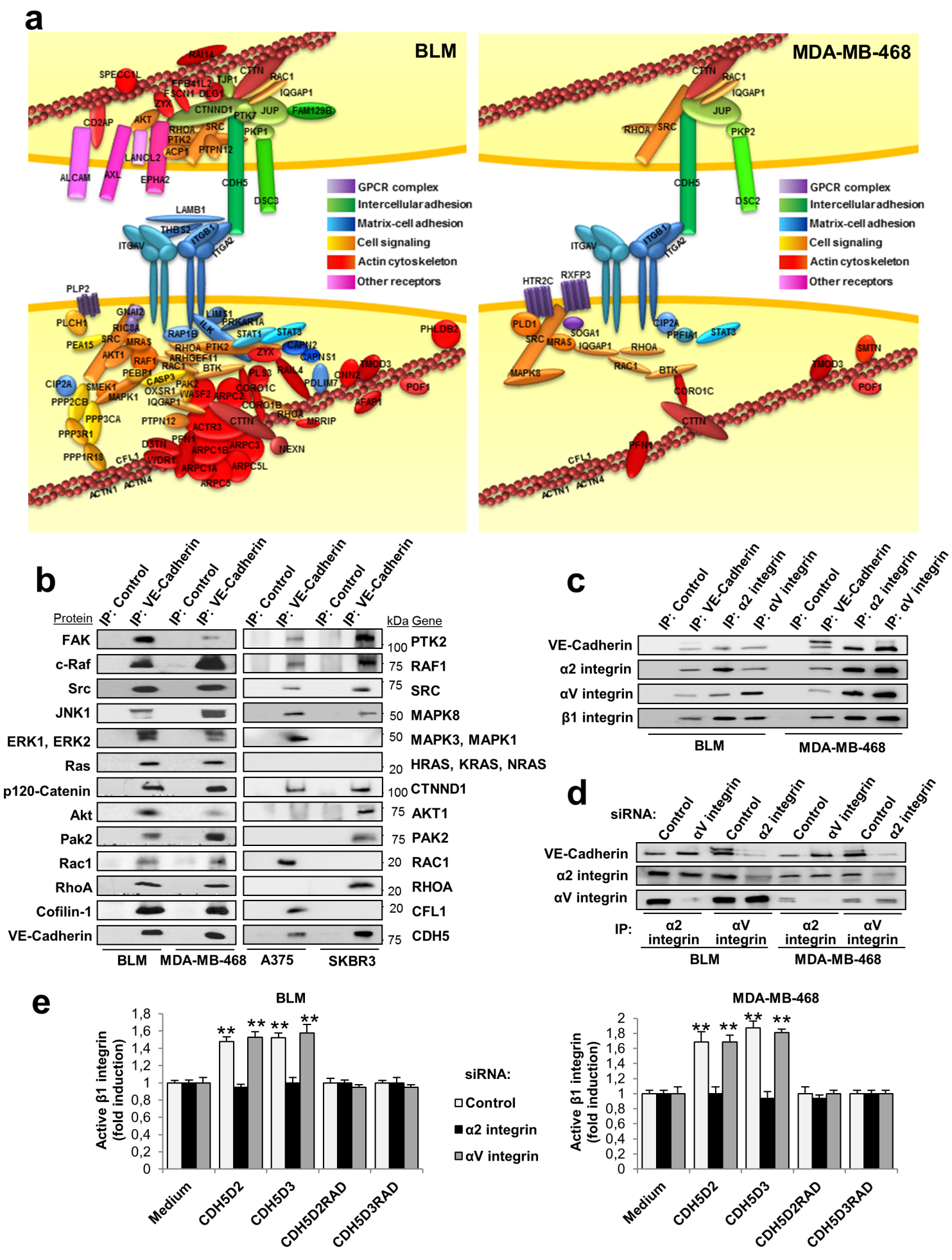

Figure 3: Protein networks identified by proteomic analyses after VE-cadherin immunoprecipitation. a. Cell lysates from BLM and MDA-MB-468 cells were immunoprecipitated using anti-VE-cadherin or control antibodies. Immunoprecipitates were resolved by SDS-PAGE, divided in three fractions and digested with trypsin. The resulting peptides were analysed by nanoLC-MS/MS. The diagram displays the proteins that were determined to specifically bind to VE-cadherin by bioinformatic analyses and data mining. b. Cell lysates from the indicated cells lines were immunoprecipitated as before, resolved by SDS-PAGE and analyzed by western blot using the indicated antibodies. c. Analysis by western blot of cell lysates from BLM and MDA-MB-468 immunoprecipitated using anti-VE-cadherin, anti- $\alpha 2$ integrin, anti- $\alpha$ v integrin or control antibodies. d. BLM or MDA-MB-468 cells were transfected with siRNAs against $\alpha 2$ integrin, $\alpha v$ integrin or control siRNAs. Cell lysates from the transfectants were subjected to immunoprecipitation using the indicated antibodies and analyzed by western blot. e. The same siRNA transfectants were exposed to the indicated peptides. $\beta 1$ integrin was significant activated by exposition to the peptides $(* * P<0.01)$. 
with RGD peptide stimulation in BLM melanoma and MDA-MB-468 breast cancer cell lines (Figure 3e). Activation of $\beta 1$ integrin by RGD peptides was blocked by $\alpha 2$ integrin silencing but not by $\alpha \mathrm{V}$ silencing in both cell lines (Figure 3e). As expected, mutant RAD peptides failed to activate $\beta 1$ integrin. In summary, the VE-cadherin interacting network consisted of $\alpha 2 \beta 1$ integrin and a large number of proteins from the integrin signalling pathway, actin cytoskeleton and cell adhesion, in addition to p120 catenin and plakoglobin.

\section{VE-cadherin signalling is involved in cell proliferation, invasion and endothelial transmigration in melanoma and breast cancer cells}

We next investigated downstream mediators of VE-cadherin- $\alpha 2 \beta 1$ integrin signalling pathway in melanoma and breast cancer cells using specific inhibitors. Integrin signalling mediates different aspects of cancer progression, including invasion and proliferation [19]. After VE-cadherin silencing, phosphorylation of SRC, ERK and JNK was significantly decreased in both cell lines, and that of AKT in BLM melanoma cells (Figure 4a). Whereas enhanced integrin signalling was observed after incubation with the RGD peptides, no changes in phosphorylation were observed after using the respective RAD mutant sequences (Figure 4a). We then tested specific inhibitors for SRC, FAK, AKT, ERK/ MEK and JNK to examine their effects on the metastatic properties, using either VE-cadherin-silenced or mocksilenced cells. None of these inhibitors affected $\beta 1$ integrin activation or cell adhesion (Supplementary Data Figure 6). However, significant effects were observed for invasion, transendothelial migration and proliferation (Figure 4b). SRC (PP2), AKT and ERK/MEK (UO126) inhibitors provoked a clear decrease in the invasive and transendothelial migration capacity of scrambled melanoma and breast cancer transfectants, similar to those achieved by VE-cadherin-silenced counterparts. No effects were observed with the FAK or the JNK inhibitors in invasion or transendothelial migration. In contrast, AKT activation in breast cancer cells was important in both, invasion and transmigration, by mechanisms VE-cadherinindependent (Figure 4a). A decrease in proliferation was observed after using PP2, UO126 and JNK inhibitors in both cancer types. Proliferation of BLM melanoma cell was also sensitive to the FAK inhibitor in a mechanism VE-cadherin-independent (Figure 4a). Inhibitors did not affect significantly to VE-cadherin-silenced cell proliferation. Collectively, these results indicate that VEcadherin promotes invasion and transendothelial migration by activation of the $\alpha 2 \beta 1$ integrin pathway through SRC and ERK. JNK activation was also implicated in cell proliferation.

\section{VE-cadherin promotes metastatic colonization in lung through its RGD motifs}

We next investigated the role of VE-cadherin and its RGD motifs in melanoma and breast cancer metastasis in vivo. We hypothesized that pre-incubation of the cells with the peptides should activate $\alpha 2 \beta 1$ integrin and, consequently, the pro-metastatic activity of the cells. For this study, we intravenously inoculated VEcadherin knockdown or wild-type control cells in mice and investigated whether lung metastasis was induced by testing for the presence of human GAPDH in lung tissue. Mice were tested at $96 \mathrm{~h}$ post-inoculation to avoid nonspecific cell binding to the lung. Lungs obtained from mice inoculated with VE-cadherin-silenced cells showed a much lower amount of the human housekeeping GAPDH gene than those inoculated with control cells (Figure 5a). In addition, lung metastasis after 5 weeks was significantly decreased in mice inoculated with VE-cadherin silenced BLM melanoma cells, compared to mice inoculated with control siRNA transfectants (Figure 5b). To confirm the pro-metastatic role of the VE-cadherin RGD motifs, we pre-incubated the cells with 9-mer peptides containing the RGD motifs in domains 2 and 3 prior to mouse inoculation. After 96 h, we noticed a significant increase in the lung colonization of melanoma and breast cancer cells, especially after using domain 3 RGD (Figure 5c). This increase in metastatic colonization was lost when the RGD peptides were replaced by the inactive RAD mutant peptides (Figure 5c). Altogether, these experiments revealed an important role of VE-cadherin with involvement of the RGD motifs in the lung metastasis of melanoma and breast cancer cells.

\section{Impact of VE-cadherin expression in patient survival}

Little is known about the impact of VE-cadherin expression on the clinical outcome of melanoma and breast cancer patients. To explore the prognostic and clinical value of VE-cadherin in patients, we performed in silico analysis of overall survival using GEO datasets. As VEcadherin is also expressed by endothelial cells, which are usually found in the tumours, we tested only samples with low expression of CD34, a marker of endothelial cells, to discard tumours with an excessive angiogenesis that might lead to confusing results. In melanoma and breast cancer, CD34 low patients with high expression of VE-cadherin exhibited a significant reduction in their survival time (log-rank $P=0.049$, log-rank $P=0.019$, respectively) (Figure 5d). Thus, high VE-cadherin expression was associated in a statistically significant manner with poorer overall survival for melanoma and breast cancer patients. In contrast, we did not find association between CD34 expression and survival (Figure 5e). These results support 
a

SIRNA:
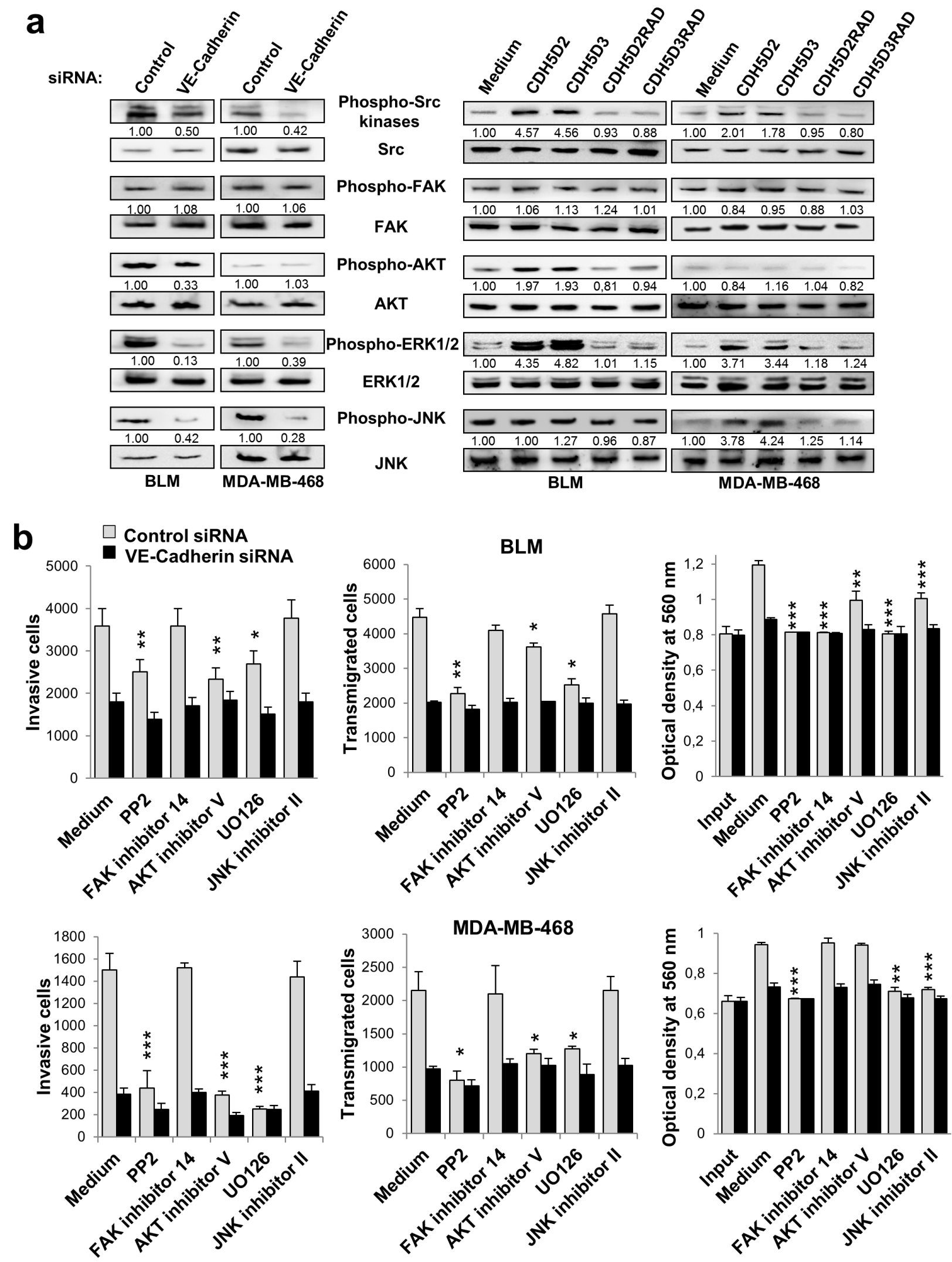

Figure 4: VE-cadherin regulates the activation of signalling proteins involved in cell invasion, transmigration and proliferation. Cancer cells were transfected with a. VE-cadherin or control siRNAs or b. exposed to the indicated peptides. Cell lysates were analyzed by western blot using the indicated antibodies. Bands were quantified using MultiGauge software. b. siRNA transfectants were tested in cell invasion, endothelial transmigration and MTT assays in presence of the indicated chemical inhibitors. 
that the correlation between VE-cadherin expression and overall survival in melanoma and breast cancer patients is not due to high vascularization of the tumours.

\section{DISCUSSION}

Here, we have demonstrated that VE-cadherin plays a major role in the induction of pro-metastatic properties - adhesion, invasion and proliferation-in different melanoma and breast cancer cell lines. We provide different pieces of evidence that the functional effects were mediated by binding of the VE-cadherin RGD motifs to $\alpha 2 \beta 1$ integrin. A model of the signalling pathway is represented in Figure 6. These results confirm the relevance of RGD cadherins in the development of cancer metastasis in multiple tumours.

a
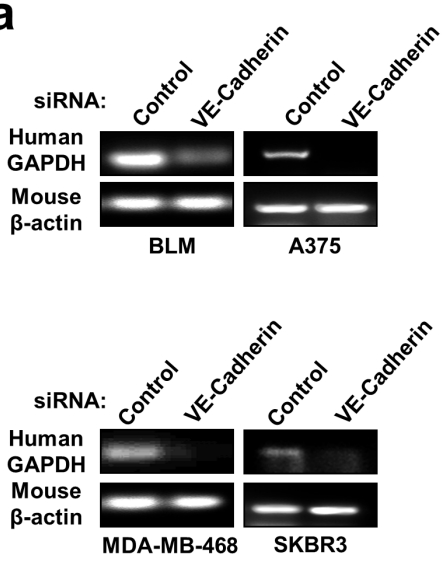

b

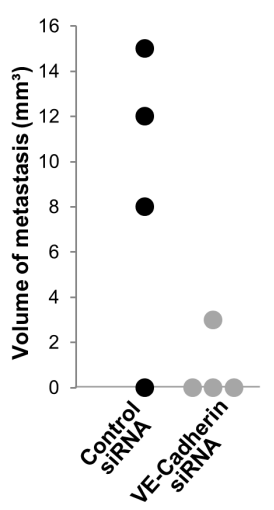

However, beyond cell specificity, there are important differences between RGD cadherins from a mechanistic point of view. For instance, VE-cadherin has two RGD motifs, which are located in domains 2 and 3 and are far from the cell surface, while $\mathrm{CDH} 17$ has one RGD, which is located in domain 7 and is quite close to the cell membrane. Moreover, while VE-cadherin contains a functional cytoplasmic domain [16], CDH17 contains a very small cytoplasmic domain not adequate for intracellular signalling. CDH17-mediated integrin activation was observed to mainly affect adhesion and proliferation in colorectal cancer [4], while VE-cadherin RGD motifs also affected invasion in melanoma and breast cancer cells. Whereas metastatic colorectal cancer cells lose their capacity to bind collagen type I and preferably bind collagen IV after $\mathrm{CDH} 17$ activation of $\alpha 2 \beta 1$ integrin [3], melanoma and breast cancer cells retained their

d
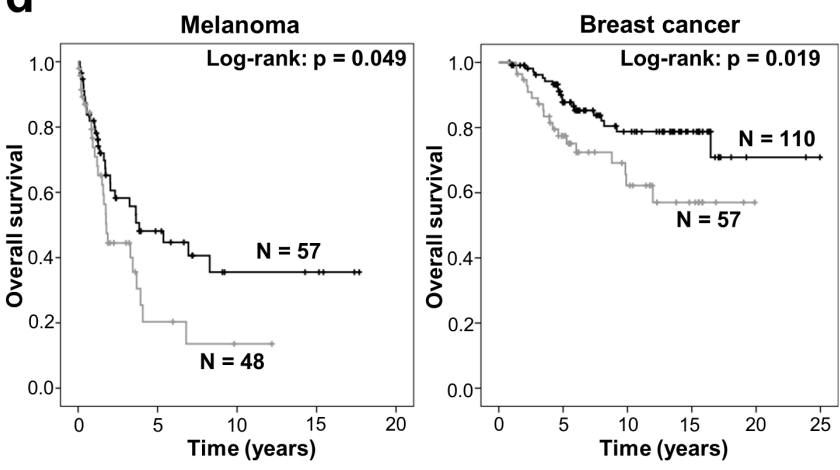

- VE-Cadherin low expression

- VE-Cadherin high expression
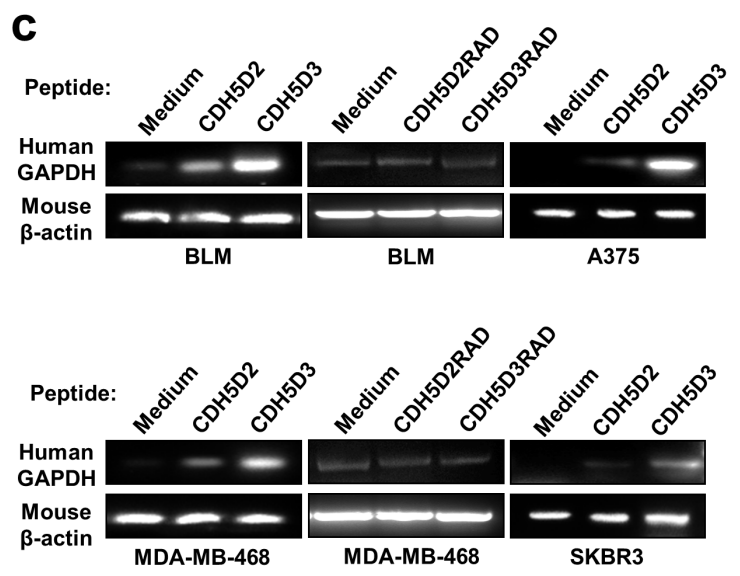

e

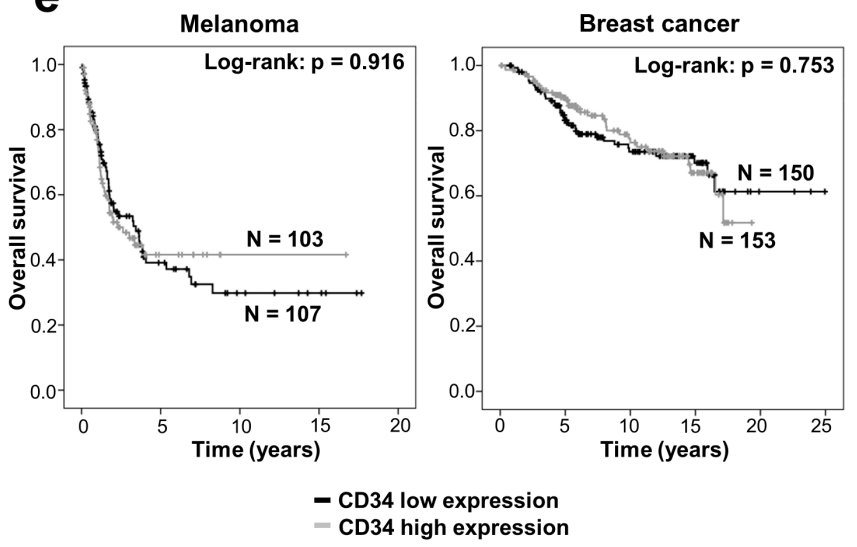

Figure 5: VE-cadherin expression is associated with lung metastasis in mouse models and poor survival in melanoma and breast cancer patients. a. BLM, A375, MDA-MB-468 or SKBR3 cells transfected with VE-cadherin or control siRNAs were inoculated intravenously in nude mice. After 4 days, mice were euthanized and lung mRNAs were isolated and subjected to RT-PCR to amplify human GAPDH. As a control, mouse $\beta$-actin was also amplified. b. BLM cells transfected with the indicated siRNAs were inoculated intravenously in nude mice $(n=4)$. After 35 days, mice were euthanized and macroscopic lung metastases were measured. $\mathbf{c}$. Cancer cells were inoculated in nude mice together with a peptide containing the RGD motifs (CDH5D2 or CDH5D3) or an irrelevant RAD sequence (CDH5D2RAD or CDH5D3RAD), as indicated. After 4 days, lung mRNAs were isolated and analysed by RT-PCR as before. $\mathbf{d}$. Significant association of VE-cadherin expression with shorter survival of melanoma and breast cancer patients was found using the logrank test. e. CD34 expression is not associated with poor survival in melanoma and breast cancer patients as found using the log-rank test. 
binding capacity to collagen I. These differences in collagen-binding capacities between both cadherins are probably due to the fact that liver parenchyma is mainly composed of collagen type IV, while lung parenchyma mostly contains type I and III collagen [20]. This is, they are related to the organ-specificity in the final metastatic colonization. In vivo experiments showed that VEcadherin silencing decreases lung colonization, while the addition of VE-cadherin RGD peptides potentiate the metastatic colonization of the lung.

It is interesting that many animal models used in cancer research, such as rodents or dogs, lack RGD motifs in their VE-cadherin sequence (Supplementary Data Figure 1). In contrast, the VE-cadherin sequence in most primates contains two RGD domains, implicating a relatively late trait acquisition that might provide some evolutionary advantage. Metastatic cells might take advantage of this evolutionary trait to improve their proliferation and invasion capacity. The lack of RGD motifs in rodent cadherins highlights that caution should be used with direct extrapolations of mouse cancer metastasis models to humans, but it also implies the existence of additional ways for metastatic colonization.

Our experiments indicate that VE-cadherin signalling does not involve $\alpha \mathrm{V} \beta 1$ integrin, which has been considered to be an RGD receptor [21], nor $\alpha \mathrm{V} \beta 3$, which was not detected. In any case, the presence of $\alpha \mathrm{V}$ in the complex deserves further investigation. In addition, we observed an interaction of VE-cadherin with p120-catenin and plakoglobin, but not with $\beta$-catenin. Therefore, VEcadherin in cancer cells keeps its cytoskeleton binding capacity (actin-binding) but does not seem to require the interaction with $\beta$-catenin for SRC or FAK activation, as it does in endothelial cells [16]. In cancer cells, SRC, ERK and JNK activation would mediate the VE-cadherin/ integrin signalling. VE-cadherin effects on proliferation of metastatic mammary cancer cells, which were previously attributed to the TGF $\beta$ pathway [14], could also be explained with the outside-in $\alpha 2 \beta 1$ integrin activation and the subsequent activation of the SRC, ERK and JNK pathways. Still, we cannot rule out that VE-cadherin might promote TGF $\beta$ activation through other indirect interactions. A correlation between higher expression of $\alpha 2 \beta 1$ integrin and higher invasiveness was previously observed in breast cancer cells [17]. RGD binding might additionally promote tyrosine phosphorylation of VEcadherin in endothelial cells, which is mediated by $\alpha 2 \beta 1$ integrin in invasive breast cancer cells [17]. In addition, tyrosine phosphorylation of VE-cadherin initiates the $\beta$-catenin dissociation from the $\mathrm{p} 120$-catenin complex to disrupt the endothelial barrier [22]. Since VE-cadherin is phosphorylated in cancer cells, it might explain why $\beta$-catenin was absent from our interaction data.

VE-cadherin is expressed also in endothelial cells and the in silico meta-analysis was carried out using RNA expression data from complete tumour tissues, which

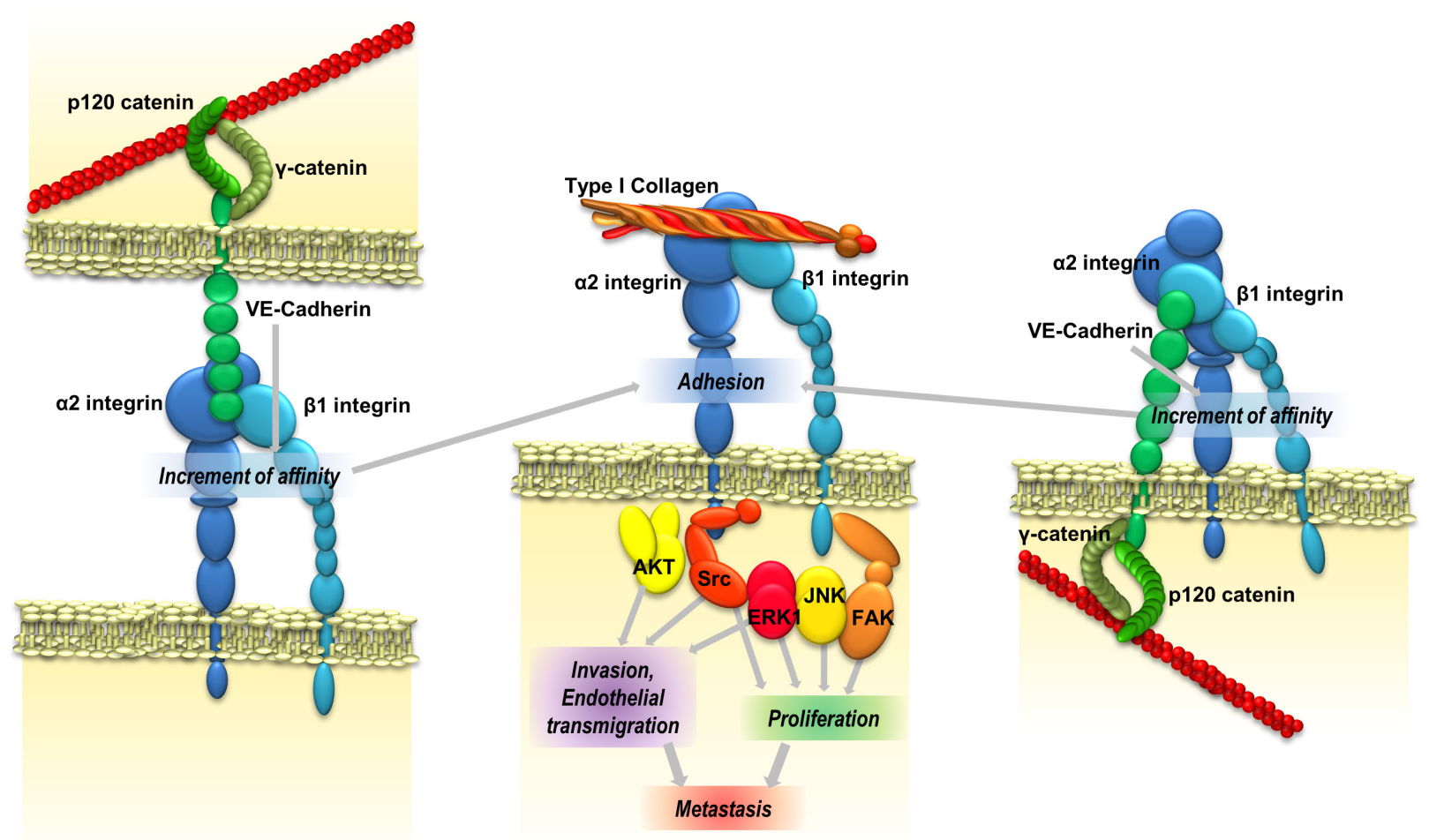

Figure 6: VE-cadherin promotes metastatic colonization. VE-cadherin RGD motifs might interact with $\alpha 2 \beta 1$ integrin either inter- or intracellularly, thereby increasing its affinity for ligands in the extracellular matrix and leading to an increase of cell adhesion and signalling activation proteins. Integrin activation increases the ability of cancer cells to invade and proliferate, and consequently their metastatic capacity. 
contain not only epithelial cells but stromal and endothelial cells. Therefore, more VE-cadherin could correlate with more angiogenesis, which in turn could finally affect the patient's survival. To clarify this point and to avoid a misinterpretation, we classified the tumours according to the expression of the endothelial marker CD34 in order to discard those tumours with high angiogenesis and vessels content from the analysis. While no association of CD34 expression with survival was observed, those melanoma and breast cancer patients who expressed high levels of VE-cadherin and low levels of CD34 showed a clear association with worst prognosis.

In summary, these studies confirm the key role of VE-cadherin and their RGD motifs in facilitating integrin activation and metastatic colonization in multiple cancer types. Interestingly, RGD motif was not required for homotypic interaction or transendothelial migration. The dissociation of the homotypic cell-cell interaction from the RGD-integrin signalling might avoid interfering with vascular stability. The enhancement of melanoma and breast cancer lung metastasis by the VE-cadherin RGD motif highlights the value of cadherin-specific RGD targeting as a novel therapeutic strategy for multiple types of cancer and metastasis. This strategy might be especially relevant for aggressive melanomas (including uveal melanoma) and breast cancer subtypes with poor prognoses, which are particularly resistant to current therapies.

\section{MATERIALS AND METHODS}

\section{Cell lines, culture and reagents}

Cells were cultured in Dulbecco's modified Eagle medium (DMEM) (Invitrogen) or RPMI (MeWo cells) containing $10 \%$ foetal calf serum (Invitrogen) and antibiotics at $37^{\circ} \mathrm{C}$ in a $5 \% \mathrm{CO}_{2}$-humidified atmosphere. Mel57 and SK-MEL-103 were gifts from Dr M. Soengas, MeWo and SK-MEL-28, from Dr P. Real (CNIO, Madrid, Spain), BLM cells, from Dr G. van Muijen (University Hospital, Nijmegen, The Netherlands), Mel-STV, from Dr. R. Weinberg (MIT, Cambridge, MA, USA) and MDAMB-468 and SKBR3, from Drs A Aranda and A Villalón, respectively (IIBB, Madrid, Spain). Cell lines A375, MDA-MB-231, MCF7 and RKO cells were purchased from the ATCC and passaged fewer than 6 months after purchase for all the experiments.

The following siRNAs were used for expression silencing: VE-cadherin, SASI-Hs01_00100751 or EHU131141 (Sigma) (assays were carried out with each one of the two siRNAs, with consistently equivalent results), integrin $\alpha \mathrm{V}$, SASI_Hs01_00220507 (Sigma), and integrin $\alpha 2$, SASI_Hs01_00123982 (Sigma). These siRNAs were transfected in tumour cells using JetPrime reagent (PolyPlus). In each transfection, an aliquot of cells was lysed and analyzed by western blot to assess VEcadherin expression silencing. Antibodies used are listed in the Supplementary Data Table S2. Nine-amino-acid peptides containing the RGD motifs from VE-cadherin were chemically synthesized in a Focus XC peptide synthesizer (AAPPTec, KY, USA). The peptides, named according to the cadherin domain where the RGD motif is found, were CDH5D2 (QGLRGDSGT, residues 233241) and CDH5D3 (SILRGDYQD, residues 296-304). As a negative control, peptides with the RGD motif mutated into an irrelevant RAD sequence-CDH5D2RAD or CDH5D3RAD - were used. The following inhibitors were used: UO126 and AKT inhibitor V (Calbiochem), PP2 (Sigma-Aldrich), JNK inhibitor II (Merck Chemicals) and the FAK inhibitor 14 (Santa Cruz Biotechnology).

\section{Flow cytometry}

Cells were detached in PBS with $2 \mathrm{mM}$ EDTA, washed and resuspended in PBS with human gamma globulin $\left(10 \mu \mathrm{g} \mathrm{ml}^{-1}\right)$ in presence of primary antibodies $\left(10 \mu \mathrm{g} \mathrm{ml}^{-1}\right)$ and incubated for $40 \mathrm{~min}$. After washing, cells were incubated in the same medium with Alexa 468-labelled anti-mouse IgG antibodies (DAKO A/S) for $30 \mathrm{~min}$. Data were analysed in a Coulter Epics XL cytofluorometer.

\section{Cell adhesion and proliferation}

Cell adhesion and proliferation assays were carried out as previously described [3].

\section{Cell invasion assays}

For invasion, $3 \times 10^{4}$ cells were resuspended in serum-free DMEM and loaded onto 8 - $\mu \mathrm{m}$ pore-size filters coated with $35 \mu \mathrm{L}$ of a 1:3 dilution of Matrigel on Transwells (Costar). The lower compartments of the invasion chambers were filled with DMEM with $5 \%$ serum, while inhibitors were added to the upper compartments. After $40 \mathrm{~h}$ of incubation at $37^{\circ} \mathrm{C}$, noninvading cells were removed from the upper surface of the filter, and invasive cells were fixed with $4 \%$ paraformaldehyde (Sigma-Aldrich), stained with crystal violet and counted under a microscope.

\section{Transendothelial migration assays}

HUVEC cells $\left(5 \times 10^{4}\right)$ were plated on $8-\mu \mathrm{m}$ poresize filter coated with fibronectin $\left(20 \mu \mathrm{g} \mathrm{ml}^{-1}\right.$; Invitrogen $)$, and confluent monolayers were incubated $16 \mathrm{~h}$ before the assay with TNF- $\beta$ (15 ng ml-1 ${ }^{-1}$ R\&D Systems). Tumour cells $\left(3 \times 10^{4}\right)$ were resuspended in serum-free DMEM, 
loaded onto the filters and incubated $24 \mathrm{~h}$. After nonmigrating cells and endothelial cells had been removed, migrating cells were fixed, stained and counted as for invasion assays.

\section{Western blot and immunoprecipitation}

Cells were detached, washed and lysed with proteases and phosphatase inhibitors in lysis buffer $(1 \%$ Igepal, $100 \mathrm{mM} \mathrm{NaCl}, 2 \mathrm{mM} \mathrm{MgCl}, 10 \%$ glycerol, 50 $\mathrm{mM}$ Tris- $\mathrm{HCl})$. Conditioned media were concentrated 10 times using Vivaspin 15R (Sartorius). Protein extracts were separated in SDS-PAGE and transferred to nitrocellulose membranes, which were incubated with primary antibodies followed by incubation with an HRP-coupled secondary antibody (Thermo Scientific) and with SuperSignal West Pico Chemiluminescent Substrate (Thermo Scientific). Densitometry analysis was performed with Quantity One software (Bio-Rad). For immunoprecipitation, cells were lysed as before, and $500 \mu \mathrm{g}$ of lysate were incubated with $100 \mu \mathrm{L}$ of protein $\mathrm{G}$ sepharose to avoid unspecific protein binding, followed by incubation with the indicated antibodies and protein $G$ sepharose beads (Sigma-Aldrich). After washing, samples were boiled and loaded on SDS-PAGE gels for western blot analysis and mass spectrometry.

\section{Mass spectrometry of immunoprecipitated proteins}

For proteomic analysis, $10 \mathrm{mg}$ of cell lysates were immunoprecipitated and the proteins were separated by SDS-PAGE, which were then divided in three slices for in-gel digestion with trypsin. Proteins were identified by mass spectrometry as previously described [4]. Identified peptides were validated using Percolator algorithm with a q-value threshold of 0.01 . Identified proteins were filtered by using the CRAPome tool to discard contaminant proteins [23].

\section{In vivo animal experiments}

For lung metastasis, $1 \times 10^{6}$ BLM, A375, SKBR3 or MDA-MD-468 cells in $0.1 \mathrm{ml}$ PBS were injected in the tail vein of Swiss nude mice (Charles River). Short term colonization assays were performed as described previously [24]. In brief, mice were euthanized 4 days after inoculation, and RNA was isolated from lungs using TRIzol Reagent (Invitrogen) and retrotranscribed. The cDNA was subjected to 30 cycles of PCR with TaqDNA polymerase (Invitrogen) to amplify human GAPDH, a housekeeping gene. For siRNA-transfected cancer cells, $1 \mu \mathrm{L}$ of the previous reaction was subjected to additional 25-cycle amplification. As a loading control, a 30-cycle amplification of murine $\beta$-actin was performed. For long term assays, mice were euthanized 5 weeks after inoculation, and macroscopic lung metastases were counted and measured.

\section{In silico prognostic studies}

Independent external cohorts of patients were used for the prognostic study. The GSE65904 database, which contains a cohort of 214 frozen melanoma samples and the GSE7390 and GSE45255 databases with 198 and 139 breast cancer samples, respectively, were used. Data were normalized using cubic spline method (melanoma) or MAS5 bioconductor (breast cancer), downloaded, and $\mathrm{Z}$-score ratios were obtained by subtracting the average $\mathrm{z}$-score for the cohort from each individual z-score and dividing the result by the standard deviation of the z-scores for the cohort, in order to remove technical variations between datasets. CD34, an endothelial marker, and VE-cadherin were independently assessed for each database. Negative and positive z-score were considered as low and high expression, respectively. Only samples with low CD34 expression were analysed for VE-cadherin expression to avoid those samples with more endothelial cells/angiogenesis. The prognostic value of VE-cadherin was assessed using Kaplan-Meier survival curves, which were plotted using IBM SPSS Statistics 2.0. Log-rank test $\mathrm{P}$ value was determined for differences in overall survival.

\section{Statistical analyses}

Data were analysed by Student's t test (2 conditions) or by one-way ANOVA followed by Tukey-Kramer multiple comparison test (more than 2 conditions). Survival curves were plotted with Kaplan-Meier technique and compared with the log-rank test. The minimum acceptable level of significance was $\mathrm{P}<0.05$.

\section{Abbreviations}

CDH17: Cadherin 17; DMEM: Dulbecco's modified Eagle medium; EMT: Epithelial-to-mesenchymal transition; EDTA: Ethylenediaminetetraacetic acid; GAPDH: Glyceraldehyde 3-phosphate dehydrogenase; HRP: Horseradish peroxidase; HUVEC: Human umbilical vein endothelial cells; PBS: Phosphatebuffered saline; PCR: Polymerase chain reaction; RPMI: Roswell Park Memorial Institute (medium); scFv: Singlechain variable fragment; SDS-PAGE: sodium dodecyl sulphate polyacrylamide gel electrophoresis; siRNA: small interfering RNA; TGF- $\beta$ : Transforming growth factor- $\beta$; TNF- $\beta$ : Tumour necrosis factor- $\beta$; VE: vascular endothelial 


\section{ACKNOWLEDGMENTS}

We thank the technical support from the CIB proteomics facility and ProteoRed platform.

\section{CONFLICTS OF INTEREST}

The authors declare no competing interests.

\section{FUNDING}

BEP was an FPI fellow from Ministry of Economy and Competitiveness (MINECO). This research was supported by grants $\mathrm{BIO} 2012-31023$ and $\mathrm{BIO} 2015-66849$ from MINECO and PRB2 (IPT13/0001-ISCIII-SGEFI/ FEDER) to JIC.

\section{Authors' contributions}

JIC conceived the project, coordinated the study, analyzed the results and wrote the manuscript. RAB conceived and conducted most of the experiments, analyzed the results and participated in the manuscript writing. ST designed and conducted the in silico analyses. SIdV and JT performed the transmigration assays. BEP and EC performed data collection.

\section{Editorial note}

This paper has been accepted based in part on peerreview conducted by another journal and the authors' response and revisions as well as expedited peer-review in Oncotarget.

\section{REFERENCES}

1. Obenauf AC and Massague J. Surviving at a distance: organ specific metastasis. Trends Cancer. 2015; 1:76-91.

2. Valastyan $\mathrm{S}$ and Weinberg RA. Tumor metastasis: molecular insights and evolving paradigms. Cell. 2011; 147:275-292.

3. Bartolome RA, Pelaez-Garcia A, Gomez I, Torres S, Fernandez-Acenero MJ, Escudero-Paniagua B, Imbaud JI and Casal JI. An RGD motif present in cadherin 17 induces integrin activation and tumor growth. J Biol Chem. 2014; 289:34801-34814.

4. Bartolome RA, Barderas R, Torres S, Fernandez-Acenero MJ, Mendes M, Garcia-Foncillas J, Lopez-Lucendo M and Casal JI. Cadherin-17 interacts with alpha2beta1 integrin to regulate cell proliferation and adhesion in colorectal cancer cells causing liver metastasis. Oncogene. 2014; 33:16581669 .

5. Dejana E. Endothelial adherens junctions: implications in the control of vascular permeability and angiogenesis. J
Clin Invest. 1996; 98:1949-1953.

6. Navarro P, Caveda L, Breviario F, Mandoteanu I, Lampugnani MG and Dejana E. Catenin-dependent and -independent functions of vascular endothelial cadherin. J Biol Chem. 1995; 270:30965-30972.

7. Carmeliet $\mathrm{P}$, Lampugnani $\mathrm{MG}$, Moons L, Breviario F, Compernolle V, Bono F, Balconi G, Spagnuolo R, Oosthuyse B, Dewerchin M, Zanetti A, Angellilo A, Mattot V, Nuyens D, Lutgens E, Clotman F, et al. Targeted deficiency or cytosolic truncation of the VE-cadherin gene in mice impairs VEGF-mediated endothelial survival and angiogenesis. Cell. 1999; 98:147-157.

8. Corada M, Zanetta L, Orsenigo F, Breviario F, Lampugnani MG, Bernasconi S, Liao F, Hicklin DJ, Bohlen P and Dejana E. A monoclonal antibody to vascular endothelialcadherin inhibits tumor angiogenesis without side effects on endothelial permeability. Blood. 2002; 100:905-911.

9. van der Schaft DW, Hillen F, Pauwels P, Kirschmann DA, Castermans K, Egbrink MG, Tran MG, Sciot R, Hauben E, Hogendoorn PC, Delattre O, Maxwell PH, Hendrix MJ and Griffioen AW. Tumor cell plasticity in Ewing sarcoma, an alternative circulatory system stimulated by hypoxia. Cancer Res. 2005; 65:11520-11528.

10. Bittner M, Meltzer P, Chen Y, Jiang Y, Seftor E, Hendrix M, Radmacher M, Simon R, Yakhini Z, Ben-Dor A, Sampas N, Dougherty E, Wang E, Marincola F, Gooden C, Lueders J, et al. Molecular classification of cutaneous malignant melanoma by gene expression profiling. Nature. 2000; 406:536-540.

11. Hendrix MJ, Seftor EA, Meltzer PS, Gardner LM, Hess AR, Kirschmann DA, Schatteman GC and Seftor RE. Expression and functional significance of VE-cadherin in aggressive human melanoma cells: role in vasculogenic mimicry. Proc Natl Acad Sci U S A. 2001; 98:8018-8023.

12. Wang L, O'Leary H, Fortney J and Gibson LF. Ph+/VEcadherin+ identifies a stem cell like population of acute lymphoblastic leukemia sustained by bone marrow niche cells. Blood. 2007; 110:3334-3344.

13. Alvero $\mathrm{AB}, \mathrm{Fu} \mathrm{HH}$, Holmberg J, Visintin I, Mor L, Marquina CC, Oidtman J, Silasi DA and Mor G. Stemlike ovarian cancer cells can serve as tumor vascular progenitors. Stem Cells. 2009; 27:2405-2413.

14. Labelle M, Schnittler HJ, Aust DE, Friedrich K, Baretton G, Vestweber D and Breier G. Vascular endothelial cadherin promotes breast cancer progression via transforming growth factor beta signaling. Cancer Res. 2008; 68:1388-1397.

15. Fry SA, Sinclair J, Timms JF, Leathem AJ and Dwek MV. A targeted glycoproteomic approach identifies cadherin-5 as a novel biomarker of metastatic breast cancer. Cancer Lett. 2013; 328:335-344.

16. Giannotta M, Trani M and Dejana E. VE-cadherin and endothelial adherens junctions: active guardians of vascular integrity. Dev Cell. 2013; 26:441-454.

17. Haidari M, Zhang W, Caivano A, Chen Z, Ganjehei L, 
Mortazavi A, Stroud C, Woodside DG, Willerson JT and Dixon RA. Integrin alpha2beta1 mediates tyrosine phosphorylation of vascular endothelial cadherin induced by invasive breast cancer cells. J Biol Chem. 2012; 287:32981-32992.

18. Jannie KM, Stipp CS and Weiner JA. ALCAM regulates motility, invasiveness, and adherens junction formation in uveal melanoma cells. PLoS One. 2012; 7:e39330.

19. Desgrosellier JS and Cheresh DA. Integrins in cancer: biological implications and therapeutic opportunities. Nat Rev Cancer. 2010; 10:9-22.

20. Suki B, Ito S, Stamenovic D, Lutchen KR and Ingenito EP. Biomechanics of the lung parenchyma: critical roles of collagen and mechanical forces. J Appl Physiol (1985). 2005; 98:1892-1899.

21. Hynes RO. Integrins: bidirectional, allosteric signaling machines. Cell. 2002; 110:673-687.

22. Chen XL, Nam JO, Jean C, Lawson C, Walsh CT, Goka E, Lim ST, Tomar A, Tancioni I, Uryu S, Guan JL, Acevedo LM, Weis SM, Cheresh DA and Schlaepfer DD. VEGFinduced vascular permeability is mediated by FAK. Dev Cell. 2012; 22:146-157.

23. Mellacheruvu D, Wright Z, Couzens AL, Lambert JP, St-Denis NA, Li T, Miteva YV, Hauri S, Sardiu ME, Low TY, Halim VA, Bagshaw RD, Hubner NC, AlHakim A, Bouchard A, Faubert D, et al. The CRAPome: a contaminant repository for affinity purification-mass spectrometry data. Nat Methods. 2013; 10:730-736.

24. Bartolome RA, Garcia-Palmero I, Torres S, Lopez-Lucendo M, Balyasnikova IV and Casal JI. IL13 Receptor alpha2 Signaling Requires a Scaffold Protein, FAM120A, to Activate the FAK and PI3K Pathways in Colon Cancer Metastasis. Cancer Res. 2015; 75:2434-2444. 